\title{
Le Franc CFA : Quel avenir pour la monnaie en zone UEMOA ?
}

\author{
SOMAN Kouadio Daouda ${ }^{1}$, SAID AHMED Djaffar Aboudou², et BENTAHAR \\ Hachemi $^{3}$ \\ ${ }^{I}$ Doctorant à l'Université Mohammed Premier, Oujda, Maroc \\ ${ }^{2}$ Doctorant à l'Université Mohammed Premier, Oujda, Maroc \\ ${ }^{3}$ Enseignant chercheur à l'Université Mohammed Premier, Oujda, Maroc
}

\begin{abstract}
Résumé : Cet article analyse la tournure de la coopération monétaire entre la France et ses anciennes colonies en cherchant à déterminer l'avenir du Franc CFA. En effet, le Franc CFA a suscité plusieurs débats et malgré les réformes institutionnelles mises en œuvre dans les années 1970, l'idée d'abandon de cette monnaie coloniale a été évoquée depuis 1983. Cependant, ce n'est que le 29 juin 2019 à Abuja (Nigéria) que le processus de la création d'une monnaie commune a été enclenché lors de la conclusion de la conférence des chefs d'Etat de la Communauté Economique des Etats de l'Afrique de l'Ouest (CEDEAO). Ainsi, «Eco » a été adopté comme le nom de la monnaie unique de la CEDEAO et devrait donc entrer en vigueur en 2020 dans les quinze États de l'espace CEDEAO. Toutefois, la mise en place de cette nouvelle monnaie ((Eco) a pris un grand retard mais son utilisation fera disparaître le Franc CFA et restreindra ou supprimera la coopération monétaire entre la France et les pays de l'UEMOA.
\end{abstract}

Mots-clés : CEDEAO, CEMAC, Eco, UEMOA, Franc CFA, Zone franc.

\section{Introduction}

La coopération monétaire, née le 26 décembre 1945 entre la France et ses colonies de l'Afrique de l'Ouest, après la ratification des accords de Bretton Woods, souffre de signes de fissuration depuis quelques années. Divers experts et citoyens de ses pays ont commencé à exprimer des critiques et à analyser les avantages et les inconvénients du Franc CFA afin réfléchir aux perspectives d'avenir. Au départ, la zone Franc regroupait toutes les colonies françaises d'Afrique subsaharienne mais une fois indépendante, certaines d'entre elles ont quitté la coopération pour introduire leurs propres monnaies. Nous pouvons citer la Guinée qui a créé sa propre monnaie en 1958, le Madagascar et la Mauritanie en 1972. Aujourd'hui, les principaux membres de la zone Franc sont la France, les pays membres ${ }^{1}$ de la

${ }^{1}$ Le Cameroun, la Centrafrique, le Congo, le Gabon, la Guinée Equatoriale et le Tchad. 
Communauté Economique et Monétaire de l'Afrique Centrale (CEMAC), les pays membres ${ }^{2}$ de l'Union Economique et Monétaire Ouest-Africaine (UEMOA) et les Iles Comores ${ }^{3}$. En dépit du fait que les deux unions (UEMOA et CEMAC) sont étroitement liées, et que leurs monnaies sont rattachées à l'euro au même niveau et partagent certaines caractéristiques institutionnelles, elles ont chacune leur propre monnaie distincte. En effet, chaque zone a un traité distinct avec la France, sa propre monnaie et ses institutions. Il y a cependant des réunions semestrielles de la zone franc CFA entre la CEMAC et L'UEMOA d'un côté et la France de l'autre. Et lors de ces réunions, les participants examinent les performances économiques dans les deux zones et décident des conceptions institutionnelles à mettre en œuvre dans les deux groupes. Mais là aussi, chaque syndicat décide des règles adéquates et des principes de fonctionnement adaptés à sa situation économique. Au niveau de l'UEMOA, l'Institut d'émission est appelée la Banque Centrale des Etats de l'Afrique de l'Ouest (BCEAO) et quant à la CEMAC, l'Institut d'émission est la Banque des Etats de l'Afrique Centrale (BEAC).

Pour rappel, le Franc CFA est une monnaie créée pendant la période coloniale et elle constitue l'une des deux institutions de la continuation du rapport postcolonial entre la France et ses anciens comptoirs d'Afrique (Nubukpo, 2007). Et après plus de 60 ans d'indépendance, cette monnaie continue d'être un intermédiaire des échanges, d'unité de compte et de réserve de valeur en Afrique francophone. Toutefois, depuis les années 1970, l'idée de réformes institutionnelles de cette monnaie ne fait qu'alimenter les débats. Alors face aux multiples revendications et contestations que sera l'avenir du Franc CFA ? Les anciens colonisateurs vont-ils accorder l'autonomie financière à leurs anciennes colonies? Le Franc CFA va-t-il disparaître au profit d'une autre monnaie?

Le but de cet article est d'abord de faire un point sur les objectifs visés lors de la création du Franc CFA, ensuite faire un état des lieux dans la pratique en examinant les différentes critiques et revendications et enfin, terminer par les avancées liées au cadre d'une nouvelle monnaie en remplacement du Franc CFA.

\section{Les principes fondamentaux du Franc CFA et les objectifs de la création du Franc CFA}

\subsection{Les principes fondamentaux du Franc CFA}

Tout accord de coopération repose sur un ensemble de principes alors la coopération monétaire entre la France et l'Afrique ne sera pas en reste. De cette manière, les mécanismes de coopération monétaire

\footnotetext{
${ }^{2}$ Le Bénin, le Burkina, la Côte d'Ivoire, la Guinée-Bissau, le Mali, le Niger, le Sénégal et le Togo.

${ }^{3}$ En dehors de la France, l'UEMOA et la CEMAC, la 'zone franc' comprend également les Iles Comores. En effet, en 1979, le gouvernement Comorien a signé un accord de coopération monétaire avec la France, faisant de Comores, une partie de la 'zone franc' mais pas de la zone franc CFA. Dans cet article, nous comptons les Iles Comores dans les discussions car l'article est basé sur le Franc CFA.
} 
entre la France et les États africains membres de zone franc composée de trois ensembles indépendants, aux monnaies distinctes, reposent sur quatre principes fondamentaux à savoir la parité fixe avec l'euro, la garantie de convertibilité illimitée du Trésor français, la centralisation des réserves de change et la libre transférabilité.

\subsubsection{La parité fixe avec l'euro}

Ce principe stipule que la parité des francs CFA doit rester fixe avec l'euro. En effet, la valeur de ces monnaies par rapport à l'euro ne change pas au jour le jour, à la différence des monnaies soumises à un régime de change flottant. Cependant, les changements de parité doivent rester exceptionnels pour ne pas porter atteinte à la confiance dans la monnaie. Ainsi, depuis 1948, la zone franc n'a connu que deux changements de parité, en 1960 et 1994 (1Franc Français, qui valait 50 Franc CFA, est passé à 100 FCFA). Et le taux d'échange n'a pas évolué lors du passage du franc français à l'euro, il s'est juste accompagné d'une conversion irrévocable entre l'euro et le franc français. Depuis 1999, un euro vaut 655,957 francs CFA.

\subsubsection{La garantie de convertibilité illimitée du Trésor français}

Selon l'article $1^{\text {er }}$ de l'accord de coopération entre la France et l'UMOA, la France ${ }^{4}$ apporte au franc CFA une garantie de convertibilité illimitée et inconditionnelle. L'objet essentiel de la garantie de convertibilité est de préserver la crédibilité de l'ancrage des francs CFA ou comorien à l'euro. En clair, cette garantie notifie que la France s'est engagée à toute demande de conversion des Banques Centrales de la Zone franc. Ainsi en cas d'épuisement des réserves de change de la Banque Centrale des Etats de l'Afrique de l'Ouest, elle pourrait se procurer des euros dont elle aurait besoin auprès de la France.

Dans la réalité, l'accord de coopération monétaire, n'est qu'un abus de langage car les accords ne se manifestent que par un engagement budgétaire de la part de la France. La garantie de convertibilité illimitée et inconditionnelle qu'apporte la France revêt en effet l'apparence d'un mécanisme de prêt, et non celle d'une garantie de l'État au sens de l'article 34 de la loi organique relative aux lois de finances.

\subsubsection{La centralisation des réserves de change}

Selon ce troisième principe, les pays de la Zone franc doivent centraliser la totalité de leurs réserves officielles auprès de leur banque centrale qui, en retour, doit obligatoirement déposer un pourcentage de ses avoirs en devises sur un compte d'opérations ouvert auprès du Trésor français. En effet, l'article 3 de l'accord de coopération entre la France et l'UEMOA, permet à la France de suivre et mesurer, à

\footnotetext{
${ }^{4}$ République Française (2004), « Décret n ${ }^{\circ}$ 2004-1362 du 8 décembre 2004 portant publication de l'accord de coopération entre le Gouvernement de la République française et les gouvernements des républiques membres de l'Union monétaire ouest-africaine, signé à Dakar le 4 décembre 1973 », Journal officiel de la République Française.
} 
travers les réserves de change, le risque financier qu'elle prend en octroyant sa garantie de convertibilité.

A la base, c'était 100\% des réserves déposées auprès du Trésor français mais ce pourcentage est passé de $100 \%$ à $65 \%$ en 1973 puis à $50 \%$ en 2005 . Cette part de réserves obligatoires déposées auprès du Trésor français doit être rémunérée par un taux de facilité de prêt marginal de la Banque Centrale Européenne (BCE) (aujourd'hui 0,25\%) actuellement. Aussi, la part des avoirs ne rentrant pas dans l'obligation de dépôt est rémunérée au taux principal des opérations de refinancement de la BCE, soit $0 \%$ aujourd'hui.

\subsubsection{La libre transférabilité}

Ce dernier principe signifie la liberté des transactions courantes et des mouvements de capitaux à l'intérieur de la Zone qui n'implique pas des transactions internes à chacune des zones monétaires (UEMOA, CEMAC). En effet, les banques centrales de chacune de ces zones peuvent instituer un contrôle de change pour les opérations avec les pays non membres de la zone monétaire. Cependant, les monnaies de la Zone franc ne sont pas librement convertibles entre elles car la France a bien conclu trois accords de coopération monétaire bilatéraux (UMOA, BEAC et Comores) mais il n'existe pas d'accord de coopération entre ces trois zones. Réellement, un franc CFA de l'Afrique de l'Ouest, utilisé par exemple en Côte d'Ivoire, ne pourra pas être utilisé au Congo (franc CFA de l'Afrique centrale).

Autre que ces quatre principes, les accords de coopération monétaire prévoient également la présence de représentants français au sein des instances techniques des banques centrales des deux zones monétaires et des Comores. Cette présence s'est fortement réduite depuis les années 1950, à l'exception des Comores où elle demeure de $50 \%$. La raison de cette présence est la garantie accordée par la France au Franc CFA.

La France était présente dans trois instances techniques de la BCEAO et de l'UMOA à savoir :

- le conseil d'administration de la BCEAO, chargé des questions relatives à la gestion de la banque centrale ;

- le conseil de politique monétaire de la BCEAO, chargé de définir la politique monétaire au sein de l'UMOA ;

- la commission bancaire de l'UMOA, chargée de la supervision bancaire.

La France ne participe plus aux instances politiques de l'UMOA (conférence des chefs d'État et de Gouvernement, conseil des ministres).

\subsection{Les objectifs de la création du Franc CFA}

${ }^{5}$ Cour des comptes, note d'analyse de l'exécution budgétaire 2019, « Gestion de la dette et de la trésorerie de l'État ». 
Après avoir amassé un flux de capitaux énormes avant la fin de la deuxième guerre mondiale, l'idée de rationaliser l'émission de monnaie dans les colonies françaises était indispensable afin de protéger les avoirs en évitant de confier l'émission aux banques locales privées (Banque de l'Algérie, Banque de l'Afrique occidentale, etc $)^{6}$. Ainsi, en signant les accords de Bretton Woods le 26 décembre 1945, la France a profité de l'occasion pour instituer le Franc des Colonies Françaises d'Afrique comme seule et unique monnaie légale des colonies. Elle effectua donc sa première déclaration de parité fixe au Fonds Monétaire Internationale ( 1 Franc CFA=1,7 Francs Français) en établissant par la même occasion, son autorité monétaire à travers la Caisse Centrale de la France d'Outre-Mer. Cependant, cette parité a subi plusieurs modifications avec le temps jusqu'au 11 janvier 1994, la date de la dévaluation du Franc CFA, soit 1 Franc CFA=0,01 Franc Français au lieu de 1 FCFA=0,02 FF précédemment. Aussi, à la création d'une monnaie unique européenne ainsi qu'à l'établissement d'une Banque Centrale Européenne et à l'arrimage du Franc CFA à l'euro, le 1er janvier 1999, 655,957 Franc CFA $=1$ euro.

Le Franc CFA, avec une parité fixe avec l'euro, devait assurer :

- La stabilité monétaire et financière : L'invariabilité du taux de change est une source de faible inflation en Zone franc par rapport au reste de l'Afrique subsaharienne. Cette stabilité préserve ainsi le pouvoir d'achat des populations. C'est pour cette raison que le taux inflation y a été inférieur à $3 \%$ au cours des dernières années tandis que la moyenne en Afrique subsaharienne est de l'ordre de $9 \%$.

- L'attractivité économique de la zone : Du fait de la parité fixe avec l'euro, les investisseurs de la zone euro ou d'autres pays sont davantage incités à investir dans de la zone franc puisqu'elle les protège contre les risques de change.

- Un facteur de solidarité et d'intégration régionale : A travers la mutualisation des réserves de change, la Zone franc favorise la définition de politiques économiques communes au sein de chaque région économique. Elle permet ainsi de créer des marchés plus importants et de favoriser une plus grande rigueur budgétaire, notamment grâce aux critères communs que les pays membres s'engagent à respecter.

Après un petit rappel de l'objectif de la création de la monnaie Franc CFA ainsi que les principes qui l'accompagnent, nous allons analyser le constat observé dans l'utilisation de cette monnaie coloniale.

\section{Constats et les revendications dans l'utilisation de la monnaie commune}

\subsection{Constats}

La Franc CFA, étant un instrument d'échange qui permet l'achat immédiat de tous les biens, services et titres sans coûts de transactions ni coûts de recherche et permet également de conserver des valeurs

\footnotetext{
${ }^{6}$ Banque de France (2015), « Note d'information : La zone franc », Eurosystème, p.2, PDF en ligne.
} 
entre deux échanges, les pays africains avaient beaucoup plus d'attente quant à l'utilisation de cette monnaie. Cependant, dans la pratique l'on a détecté des avantages et des inconvénients que nous allons essayer d'énumérer certains.

\subsubsection{Les privilèges de la Zone Franc CFA pour ses pays membres}

Le Franc CFA a su maintenir la stabilité monétaire au sein des régions qui l'utilisent et il a beaucoup contribué à l'intégration régionale.

L'arrimage à une monnaie stable comme l'euro, en plus de sa libre convertibilité, occasionne des gains microéconomiques en réduisant l'incertitude relative aux fluctuations du taux de change, renforçant la crédibilité du franc CFA et favorisant en théorie l'afflux de capitaux étrangers. Aussi, elle stimule théoriquement la croissance à travers l'intégration commerciale due à l'utilisation d'une monnaie commune par les pays membres qui permet la suppression des coûts de transaction et du risque de change lors des échanges intra-zone.

En effet, une monnaie stable préserve les pays utilisateurs de violents de change et ces derniers peuvent revaloriser les dettes et produits d'importations libellés en monnaie étrangère. Elle accomplit efficacement ses fonctions de moyen d'échange, d'unité de compte et de réserve de valeur, en raison de la stabilité de son pouvoir d'achat. Il faut notifier qu'à travers les statuts de la Banque de France, la parité fixe s'accompagne d'une surveillance étroite de la politique monétaire des Banques Centrales du Franc CFA, faisant abstraction de toute politique monétaire de propagation. Cela freine diligemment l'inflation dans les pays membres. En ce sens, nous pouvons ajouter qu'une monnaie est dite stable lorsque le niveau général des prix, mesuré par l'indice des prix à la consommation, ne varie pas trop.

L'inflation altère clandestinement la valeur réelle des contrats et de l'épargne en compromettant non seulement la franchise mais aussi à l'efficacité économique. En effet, elle engendre une incertitude et une perte de confiance qui pèse sur l'investissement, sur l'activité économique et sur l'emploi. Les entreprises rencontrent des difficultés dans la prévention de leurs prix et leurs coûts, dans la planification de leurs investissements et dans la prise de bonnes décisions. Aussi, les prêteurs exigent des taux d'intérêt plus élevés, comportant une prime de risque d'inflation. Heureusement, les pays de la Zone Franc sont épargnés de cette situation. Ces pays ont plus ou moins une maitrise sur l'inflation ce qui engendre une protection ${ }^{7}$ du pouvoir d'achat des populations, une attractivité renforcée pour les investisseurs privés et les bailleurs internationaux, une stabilité du cadre macroéconomique et le développement à long terme des systèmes financiers.

Il est important de noter qu'avant l'arrivée de la pandémie de covid-19, l'activité économique de 1'UEMOA enregistrait une croissance soutenue depuis 2012. En effet, en 2019, la Zone a enregistré un

${ }^{7}$ Feindouno S., Guérineau S., Guillaumont P., Sylviane Guillaumont J. S, et Plane P. (2019), «Zone franc, croissance économique et pauvreté », Edition Ferdi. 
taux de croissance global de 6,1\%, bien supérieur à la moyenne d'Afrique Subsaharienne, et dans un contexte de stabilité des prix. Quant au déficit budgétaire global de l'Union, il s'est réduit à 2,3\% du PIB, contre 3,4\% en 2018. Aussi, le taux d'endettement de l'UEMOA est resté limiter à 44,5\% du PIB en décembre 2019. Vu ces chiffres, on peut dire que les équilibres macroéconomiques étaient partant satisfaisants.

Toutefois, malgré la pandémie, l'économie de l'UEMOA demeure l'une des économies les plus dynamiques de l'Afrique Subsaharienne et du monde. Selon $\mathrm{FMI}^{8}$, la croissance de l'Union s'établirait à 2,3\% en 2020 contre 6,3\% prévu auparavant. Ainsi, malgré la crise, à l'exception de la GuinéeBissau, le FMI prévoyait que les pays de l'Union devraient tous connaître des taux de croissance positifs en 2020, sous réserve d'une dégradation ultérieure : Burkina Faso (+2\%), Côte d'Ivoire $(+2,7$ $\%)$, Mali (+1,5\%), Niger (+1\%) ou Sénégal (+3\%).

En somme, la stabilité du taux d'échange participe à une inflation plus faible dans la Zone Franc comparée au reste de l'Afrique Subsaharienne, en épargnant par la même occasion le pouvoir d'achat des populations. Au sein de cette zone, l'inflation a été inférieure à $3 \%$ au cours des dernières années quand la moyenne en Afrique Subsaharienne est de l'ordre de 9\%.

\subsubsection{Les insuffisances du Franc CFA}

Le Franc CFA a pour objectif d'assurer la stabilité monétaire et financière, l'attractivité économique puis favoriser la solidarité et l'intégration régionale de la Zone. Si dans l'ensemble, quelques objectifs semblent être atteints, il reste cependant beaucoup de choses à déplorer.

L'arrimage du Franc CFA à l'Euro impose une cible d'inflation de 2\% comme principal objectif de la politique monétaire de l'UEMOA. Cependant, dans un régime de parité fixe avec une libre circulation des capitaux, il est incompatible pour les Banques Centrales des pays membres de disposer d'une politique monétaire durablement différente de la zone ancre, c'est-à-dire la zone Euro ${ }^{9}$. Ainsi, la BCEAO rencontre des difficultés en ce qui concerne le choix de son objectif de politique monétaire. Aussi, dans ce type de régime, du fait de la perte de compétitivité qu'entraine l'inflation, elle participe à la détérioration progressive des comptes extérieurs et à la surévaluation du taux de change, affaiblissant ainsi la parité entre les monnaies.

Dans les normes, l'adoption d'une monnaie commune est une étape importante dans le processus d'intégration car elle fait appelle à une coopération et/ou une coordination d'un ensemble plus large de politiques économiques et monétaires. Un processus qui pourrait aboutir à une convergence des politiques nationales sensées engendrer des économies d'échelle et des effets spillovers positifs comme la construction d'un marché commun, le lancement des projets d'investissements ou une

${ }^{8}$ Fond Monétaire Internationale (2020), « World Economic Outlook », www.imf.org.

${ }^{9}$ Mundell R. A. (1961), « A theory of optimum currency areas », American Economic Review, Vol. 51, No. 4 pp. 657-665. 
meilleure surveillance multinationale d'où un projet de libre circulation des biens et des personnes dans la Zone. Or en réalité, après plus de 75 ans d'existence, on note toujours un faible volume d'échanges intracommunautaires ${ }^{10}$ soit $15 \%$ de l'ensemble des échanges contre $60 \%$ au sein de la Zone euro.

Parmi les insuffisances de cette monnaie, nous notons aussi, la faible compétitivité prix à l'exportation généralement due au mésalignement du taux de change réel du franc CFA par rapport à l'euro. A travers une étude ${ }^{11}$ basée sur la sous-évaluation ou la surévaluation réelle du Franc CFA réalisée sur la période de 2001-2011, les estimations économétriques ont montré une différence entre les Taux de Change Effectif Réel (TCER) et les TCER observés. Les résultats de cette étude ont confirmé le mésalignement du Franc CFA en 2011 calculé pour chaque pays africain de la Zone franc suivant la méthode d'Elbadawi et Soto (1997). On a également constaté que le TCER était surévalué dans certains pays par contre il était sous-évalué dans d'autres. Ce qui pousse à dire que les situations sont très différentes d'un pays à un autre même au sein du CEMAC ou UEMOA et donc les politiques devraient être différenciées.

Egalement, le rationnement endogène du crédit qui représente que 23\% du PIB (crédits à l'économie rapportés au PIB) contre $100 \%$ au sein de la zone euro, constitue un frein au développement des pays membres de la Zone. Selon Baltensperger (1978), le rationnement de crédit est une situation de rejet de demande de financement de projet même si l'emprunteur est prêt à payer des taux d'intérêt élevés et fournir des garanties importantes. Et pourtant, les banques centrales sont supposées venir emprunter auprès du Trésor français en contrepartie des $50 \%$ des réserves de change placées sur un compte d'opération en son sein comme le stipule les termes de l'accord entre le Trésor et les Banques Centrales. D'ailleurs, pour Michel Abdelouhab, cette partie des réserves de change représente «autant de ressources en moins pour le financement du développement économique ».

Certaines actions du fonctionnement de la monnaie unique qui jusqu'à aujourd'hui sont restées identiques rattachent les pays de la Zone à leur colonisateur. Il est question du fait que les billets et les pièces de la monnaie CFA sont toujours exclusivement fabriqués en France avec de surcroit, des frais de tirages, ce qui pousse certains citoyens à douter de la souveraineté des pays de la Zone Franc. Un constat malheur est que malgré l'indépendance des pays de Zone Franc, la BCEAO et la BEAC doivent toujours passer par le Trésor français pour accéder à la garantie de la parité fixe entre le Franc CFA et l'euro, et non par leur consœur de Francfort, la Banque centrale européenne, comme on aurait pu s'y attendre.

\footnotetext{
${ }^{10}$ Jeune Afrique (2016, 21 Oct.), « De quoi le franc CFA est-il le nom ?», www.jeuneafrique.com.

${ }^{11}$ Gnansounou S. U. et Verdier-Chouchane A. (2012), « Mésalignement du taux de change effectif réel : Quand faudra-t-il de nouveau dévaluer le franc CFA ? », Banque Africaine de Développement, Working Paper series, N 166- Décembre 2012.
} 
Enfin, les deux principales banques centrales (BCEAO et BEAC) ne poursuivent qu'un seul et unique objectif, celui de la stabilité des prix.

En bref, la stabilité monétaire, le faible taux d'inflation et l'absence de risque de change semblent incomplets pour un continent à forte croissance démographique avec un niveau de développement économique très insuffisant. Les besoins de la zone sont énormes et peuvent se décliner en création d'emplois, d'infrastructures de base encore déficientes (santé, éducation, transport, etc.) ... Ainsi, face à ses insuffisances, l'opinion publique ne s'est pas fait attendre.

\subsection{Les revendications dans l'utilisation de la monnaie commune}

L'idée d'obtenir plus de visibilité ou son indépendance monétaire est née au cours des années soixante-dix. En effet, les Etats membres ont exprimé pour la première fois leur désir qui s'est concrétisé par l'adoption des réformes après la signature de nouveaux accords de coopération monétaire entre 1972 et $1973^{12}$. Depuis cette époque, la présence de la France au sein des conseils d'administration de la BCEAO et la BEAC s'est vue réduite. Dans le même temps, le siège des Banques centrales est transféré en Afrique en 1977 pour la BEAC (Yaoundé) et en 1978 pour la BCEAO (Dakar). Ainsi, les postes de responsabilité dans les services centraux et les directions nationales de chaque banque centrale sont progressivement confiés à des cadres ressortissants des pays membres.

Malgré ces réformes, l'existence de Zone franc faisait toujours débat dans plusieurs conversations jusqu'à ce qu'on enregistre des publications et des manifestations dans lesquelles certains exigent des réformes plus approfondies pendant que d'autres exigent la création d'une nouvelle monnaie sans l'ingérence de la France. En effet, plusieurs groupes de populations ont pris part à l'expression des insuffisances du Franc CFA à savoir des économistes et auteurs d'ouvrages, la société civile africaine, la diaspora ...

\subsubsection{Certains économistes et auteurs des ouvrages}

En 2016, Kako Nubukpo, ancien ministre de la prospective du Togo et ancien directeur de la francophonie économique et numérique au sein de l'Organisation internationale de la francophonie, et ses collègues ont écrit l'ouvrage 'Sortir l'Afrique de la servitude monétaire : à qui profite le franc CFA ? Cet ouvrage, le plus récent d'ailleurs, réunit dix auteurs, économistes africains et français. Il rassemble à lui seul, la synthèse des arguments défendus par des auteurs souhaitant des réformes et appelant à des progressions des dispositions jugées problématiques des conventions de la Zone et celle des arguments soutenus par des auteurs désirant l'abolissement et prêts à sortir de la Zone pour une expérience monétaire sans les anciens colonisateurs. Cet ouvrage a véritablement contribué au

\footnotetext{
${ }^{12}$ Banque de France (2015), « Note d'information : La zone franc », Eurosystème, PDF en ligne.
} 
mouvement évolutionniste car il a révélé certains aspects de la Zone franc qui étaient jusque-là, ignorés par plusieurs personnes.

Au sein de cet ouvrage, certains auteurs comme Kako Nubukpo, Martial Ze Belinga et autres, attestent que le fait que les anciennes colonies françaises en Afrique, partagent la même monnaie, le franc CFA, constitue un instrument de répression politique aux mains de l'ancien colonisateur, la France, puis représente un handicap au développement des pays utilisateurs. Aussi, Nadim Michel Kalife, après avoir rappelé la genèse de la monnaie, il a insisté sur le 'pacte colonial'. Selon ce pacte, les économies des pays de la Zone franc, c'est-à-dire les pays ayant en partage l'utilisation du Franc CFA, devaient être cantonnés uniquement au rôle de pourvoyeuse de matière à la métropole, cette dernière se chargeant de les exporter ou de les transformer grâce à son tissu industriel. Partant de cette vision, le Franc CFA n'avait pas pour ambition de permettre formellement la compétitivité de l'économie des pays qui l'utilisent. Ainsi, le Franc CFA apparait à la lumière de son histoire et de ses mécanismes de fonctionnement, comme non seulement un reliquat colonial mais aussi et surtout comme un véritable frein à tout dynamisme de l'économie, et ceci justement en raison du fait que sa dimension politique est occultée. Pendant que pour certains auteurs, le franc CFA est une 'servitude volontaire' ${ }^{13}$, pour d'autres cette monnaie représente l'image de la main coloniale, empêchant une réelle indépendance des États du pré carré français. C'est dans ce sens qu'intervient le sixième chapitre de l'ouvrage en invitant à rompre obligatoirement la tutelle coloniale pour parachever les indépendances en se servant d'une politique de développement et progrès social dont le lanceur serait la souveraineté monétaire des pays de la zone franc.

Pour finir, l'ouvrage convie ces pays à prendre leur destin monétaire en mains, avec les risques économiques afférents, en rompant notamment avec le système du franc CFA. Toujours, pendant que certains auteurs exigent une nécessité d'une sortie pure et simple de la zone franc en mettant les dirigeants devant leurs responsabilités, d'autres recommandent à ces Etats de tirer parti des avantages des marges de manœuvre encore largement sous exploitées par les banques centrales africaines telles que l'arrimage à un panier de devises ${ }^{14}$ plutôt qu'uniquement à l'euro sous le couvert des institutions françaises, un ciblage moins rigide de l'inflation, la prise en compte de la croissance etc. Ces idées évolutionnistes ont encouragé les activistes et ont contribué fortement à l'amélioration de la situation monétaire en Afrique subsaharienne.

\footnotetext{
${ }^{13}$ Nubukpo K. (2007), « Politique monétaire et servitude volontaire. La gestion du franc CFA par la BCEAO », Politique africaine, $\mathrm{n}^{\circ} 105,2007$, p. 11.

${ }^{14}$ Carlos Lopez, l'ancien secrétaire général adjoint de l'ONU, alors à la tête de la Commission économique de l'ONU pour l'Afrique, critiquait comme désuète la convertibilité à taux fixe du franc CFA et plaidait pour un franc CFA plus fluctuant, arrimé par exemple à un panier de devises.
} 
A cet ouvrage, il faut ajouter plusieurs publications, colloques ouverts et émissions grand public animés par l'économiste togolais Kako Nubukpo qui ont joué un rôle très important dans le mécanisme de changement de monnaie.

\subsubsection{La société civile africaine et la diaspora}

La société civile a aussi contribué au mouvement relatif à la modification des reformes ou à l'abandon du FCFA et parmi les personnes les plus remarquées dans cette voix, nous avons KEMI SEBA, président de l'ONG Urgences Panafricanistes qui est allé jusqu'à bruler un billet de 5000 FCFA le 19 Août 2017 à la place de l'Obélisque à Dakar au Sénégal. En effet, l'activiste franco-béninois connu pour ses propos controversés, réclame juste une indépendance monétaire des Etats africains à travers un geste symbolique pour lui. Selon lui, au $\mathrm{XXIe}^{15}$ siècle, chaque peuple a le droit de posséder sa propre monnaie et aucun avenir politique ne peut se décider sans une emprise totale sur son économie. Or jusqu'aujourd'hui, la Banque de France a toujours le droit de dire si oui ou non elle est d'accord par rapport aux décisions économiques que prennent les pays de la zone franc. Pour lui, cela est une preuve suffisante que le franc CFA est une monnaie caduque, d'esclavagisme et de soumission. A cause de son acte, l'activiste Kémi Séba a été arrêté à Dakar le vendredi 25 août, à 7 heures du matin, pour le placer en garde à vue ${ }^{16}$. La Banque Centrale des Etats de 1'Afrique de l'Ouest (BCEAO) avait porté une plainte contre lui pour le billet colonial brulé. Pour la Banque, certes la valeur inscrite sur un billet de banque appartient au détenteur de ce dernier, mais le billet de banque, dans sa matérialité et comme support de cette valeur, appartient à son émetteur, la Banque Centrale. Détruire un signe monétaire, dans le droit sénégalais, est une infraction. Néanmoins, Ils ont été acquittés ${ }^{17}$, lui et son soit disant complice par un tribunal de Dakar le 29 août et il a été expulsé du Sénégal vers la France le 6 septembre 2017.

Arrivée en France, Kémi Séba continue sa lutte, il s'est même rendu à Rome pour rencontrer des cadres du Mouvement 5 étoiles, dont le secrétaire d'État aux Affaires étrangères, Manlio Di Stefano. Ce dernier soutient la lutte de l'activiste et se déclare prêt à les aider en fournissant des arguments qui ont été au centre d'un incident diplomatique entre la France et l'Italie. Aussi, après avoir eu ce soutient, le même jour, Luigi Di Maio, président du M5S et vice-président du Conseil italien ${ }^{18}$, lance une

\footnotetext{
${ }^{15}$ Christinaz C. (2017, 04 Sept.), «Les francs CFA brûlés par Kémi Séba enflamment les réseaux », journal le temps, www.letemps.ch.

${ }^{16}$ Bouessel du Bourg C. (2017, 29 Août), « Franc CFA : l'activiste Kémi Séba arrêté à Dakar pour avoir brûlé un billet de banque », Journal jeune Afrique, www.jeuneafrique.com.

${ }^{17}$ Niasse Ba S. (2017, 07 Sept.), « Le Sénégal a expulsé vers la France le militant suprématiste noir Kémi Séba », Journal le Monde, Afrique, www.lemonde.fr.

${ }^{18}$ Licourt J. (2019, 04 Fév.), « Kémi Séba, sulfureux conseiller du gouvernement italien », le figaro, International, www.lefigaro.fr.
} 
violente charge contre Paris, accusée «d'empêcher le développement» des pays utilisant le franc CFA. Il ajoute que la France «contribue aux départs des migrants, qui vont ensuite mourir dans la Méditerranée ou débarquer sur nos côtes ». Il est temps que l'Europe ait le courage d'aborder le thème de la décolonisation de l'Afrique». Pour l'Italie, avec le franc CFA, la France maintient ses colonies en Afrique et aussi lors du sommet Russie-Afrique de Sotchi, les russes ont clairement pris fait et cause pour les anti-CFA.

Toujours dans la même année 2017, plusieurs centaines de manifestants se sont réunis dans plusieurs villes d'Afrique francophone telles que Cotonou, Dakar, Libreville, Bamako, à l'appel du mouvement Urgences panafricanistes, pour dire non au franc CFA, qui selon eux "empêche le développement", sur fond d'une polémique qui gagne notamment le Sénégal et le Bénin ${ }^{19}$.

Selon l'Agence France-Presse (AFP), Simon Kouka, responsable de groupe de société civile sénégalaise Y'en a marre a déclaré : "On ne peut pas se dire souverains et dépendre d'une monnaie comme le franc CFA», «la souveraineté implique avoir sa propre monnaie». A ses déclarations s'ajoute celle de Keeman Diouf qui affirmait que «Le franc CFA nous empêche de nous développer » et il se présente comme artiste et ouvrier. Il a fait sensation en avalant un billet de 500 francs CFA.

Toutefois, la création d'un Conseil Présidentiel pour l'Afrique le 29 Août $2017^{20}$ dont le rôle est de remonter les souhaits des sociétés civiles africaines du continent et de ses diasporas, a beaucoup contribué à l'abandon du FCA. En effet, il s'agit de donner un nouveau visage à la relation entre l'Afrique et la France à travers sa composition paritaire rassemblant des personnalités de France et d'Afrique issues de la société civile dont la mission est d'apporter un éclairage nouveau à la politique du Président de la République Française sur l'Afrique. Ainsi, le Conseil Présidentiel pour l'Afrique représente une innovation tant sur la forme, en renouvelant le mode de gouvernance traditionnel de la politique extérieure de la France en Afrique, que sur le fond, en apportant une matière nouvelle tournée vers l'avenir et vers les attentes des jeunesses africaines et françaises. Ce conseil a facilité le dialogue entre l'Afrique et la France sur plusieurs sujets importants.

Il y a aussi l'intérêt porté par le Fonds Monétaire International ${ }^{21}$ au Franc FCA depuis sa première dévaluation.

\footnotetext{
${ }^{19}$ Le Monde et AFP (2017, 18 Sept.), « Manifestations d'opposants au franc CFA en Afrique et en région parisienne », le Monde, www.lemonde.fr.

${ }^{20}$ www.cpafrique.fr.

${ }^{21}$ Diawara M (2016, 24 Déc.), « Cémac : pas de dévaluation du franc CFA, mais des aménagements nécessaires », Journal le Point, www.lepoint.fr.
} 
A cela s'ajoute une poussée de la Chine sur le plan économique, mais aussi monétaire en Afrique ${ }^{22}$. Cette poussée s'est traduite par un fort endettement des Etats africains vis-à-vis de la Chine et ils ont été obligés de négocier avec le Fonds Monétaire International (FMI) pour des remises de dettes. En cinq ans seulement, les investissements directs cumulés par la Chine dans les pays concernés ont dépassé 60 milliards de dollars et la valeur des projets signés par des entreprises chinoises a atteint plus de 500 milliards, l'institution de Bretton Woods précise que ces partenariats peuvent, du fait de leur impact sur le niveau d'endettement, limiter les moyens et dépenses vers certains autres fronts prioritaires en raison des frais liés aux intérêts de ces prêts. Ainsi, avec une forte dette, le 15 août 2016, l'Angola a adopté la monnaie chinoise ayant cours légal et pouvoir libératoire sur tout son territoire. De même, en août 2018, le Nigeria aussi a passé un accord avec la Chine permettant de contourner le dollar et l'euro pour faire des transactions entre le yuan chinois et le naira nigérian. Cette manière de contourner le dollar ou l'euro pour des transactions rendait les monnaies de ses pays internationales ce qui n'était pas vraiment le cas avec le Franc CFA et qui la rendait comme une sorte de réplique coloniale.

Tous ces débats, meetings, conférences, publiques, publications ... ont fini par donner leur fruit. Cependant, plusieurs rencontres ont eu lieu entre les dirigeants des pays concernés et aussi, entre les dirigeants de ces mêmes pays et la France. A l'insu de ces rencontres plusieurs réformes ont été prises qui vont jusqu'à changer le Franc CFA en l'éco en théorie.

\section{Modifications : les réformes du Franc CFA et la nouvelle monnaie}

\subsection{La nouvelle monnaie de la zone CEDEAO}

Après les conclusions de la conférence des chefs d'Etat de la Communauté Economique des Etats de 1'Afrique de l'Ouest (CEDEAO) le 29 juin 2019 à Abuja (Nigéria) ${ }^{23}$, le processus de la création d'une monnaie commune a été enclenché. En effet, «Eco»a été adopté comme le nom de la monnaie unique de la CEDEAO et devrait donc entrer en vigueur en 2020 dans les quinze États de l'espace CEDEAO. Ce n'est pas un projet récent, il a été reporté à maintes reprises depuis 1983.

Toutefois, plusieurs étapes restent à affranchir et qui ne se réaliseront pas sans difficultés majeures. D'abord, huit pays devront abandonner le Franc CFA tandis que sept autres devront abandonner leur monnaie nationale. Aussi, il a été décidé que les pays respectant d'office les critères de convergences (ceux de l'UEMOA) débutent avec la monnaie unique en attendant que les autres s'alignent. En effet, les principaux critères sont :

\footnotetext{
${ }^{22}$ Diawara M. (2019, 07 Mai), « Dette et colonisation de l'Afrique : ces peurs que réveille la Chine », Journal le Point, www.lepoint.fr.

${ }^{23}$ Jeune Afrique et AFP (2019, 02 juillet), « « Eco », future monnaie unique de la CDEAO : les chefs d'État maintiennent l'objectif de $2020 »$, Journal jeune Afrique, www.jeuneafrique.com.
} 
- un déficit budgétaire inférieur à $3 \%$ du PIB ;

- une inflation inférieure à $10 \%$;

- un ratio Dette/PIB inférieur à $70 \%$.

Cette convergence économique a été pendant longtemps un frein à la création de la monnaie unique. L'une des difficultés est que la CEDEAO est une organisation qui a un profil monétaire particulier car elle est constituée de huit Etats $^{24}$ formant l'Union Economique monétaire ouest-africaine (UEMOA) qui utilise le franc CFA, autrefois arrimé au franc français et aujourd'hui à l'euro tandis que les sept autres pays ${ }^{25}$ ont chacun leur monnaie.

Une autre difficulté est que les sept autres pays craignent que leurs souverainetés monétaires soient compromises par une ingérence française, par le biais des pays de la zone franc CFA. Pour le Nigeria, le géant sous-régional, la CEDEAO doit éviter toute précipitation dans le processus de mise en place de la future monnaie. Il affirme que «Si nous prenons une décision sur notre monnaie, elle doit être prudente, pour ne pas mettre notre économie en difficulté. C'est très important et c'est pourquoi les responsables de notre banque centrale ont été très précautionneux sur la question $»^{26}$, confiait Musa Nuhu, le chef de la cellule nationale CEDEAO au ministère nigérian des Affaires étrangères dans les colonnes du journal nigérian Vanguard en mai 2019. En parlant des pays francophones qui utilisent le Franc CFA, il déclare que : «Le problème qui se pose, c'est que la Banque centrale du Nigeria leur demande de dissocier le CFA de la Banque de France. La valeur de ce franc CFA est déterminée par la Banque de France, qui conserve leurs réserves et fixe également son taux de change.» Or pour le naira (la monnaie nigériane), «nous ne nous cachons pas derrière les institutions (...) nos réserves sont en dollars américains, en euros et récemment en (yuan) chinois, nous sommes donc seuls. Si une monnaie se cache derrière une autre, on ne peut pas apprécier sa valeur réelle».

Il est vrai que le nom est d'ores et déjà connu et ratifié lors de ce sommet d'Abuja mais le symbole et l'iconographie qui apparaîtront sur les billets ne font pas encore consensus. Et cette monnaie admet plusieurs caractéristiques différentes du Franc CFA. Contrairement au Franc CFA, le taux de change de l'éco sera flexible et la monnaies évoluera en fonction d'un panier de devises. Pour atteindre les objectifs de création et de suivi de cette monnaie, une banque centrale fédérale doit voir le jour.

$\mathrm{Au}$ vu des multiples préoccupations dont la CEDEAO doit résoudre avant la mise en circulation de cette monnaie unique, la zone UEMOA, en accord avec la France, ont procéder à des réformes du Franc CFA. On peut dire que ces réformes peuvent faciliter la mise en place de l'éco car elles

\footnotetext{
${ }^{24}$ La CEDEAO est constitué de huit Etats de l'UEMOA qui sont Bénin, Burkina Faso, Côte d'Ivoire, GuinéeBissau, Mali, Niger, Sénégal et Togo.

${ }^{25}$ Les sept autres pays de la CEDEAO : le Cap-Vert dont la monnaie a aussi une parité fixe avec l'euro, la Gambie, le Ghana, la Guinée, le Liberia, le Nigeria et la Sierra Leone.

${ }^{26}$ Gbadamassi F. (2019, 30 juin), «CEDEAO : en attendant la monnaie unique... », Journal franceinfo Afrique, économie africaine, www.francetvinfo.fr.
} 
suppriment quelques inquiétudes des sept autres pays en matière de l'ingérence de la France. Surtout le Nigeria qui a exigé que les pays de l'UEMOA rompent tout lien avec le Trésor français avant la concrétisation de l'éco, c'est désormais chose faite avec l'annonce de la fin du franc CFA. Cette décision a créé une convergence des intérêts pour chacun des pays de la région ouest-africaine.

\subsection{Les réformes du Franc CFA}

Le 21 décembre 2019, le Président ivoirien Alassane Ouattara et le Président français Emmanuel Macron ont communiqué une réforme des relations de coopération monétaire entre la France et l'Union Economique et Monétaire Ouest-Africaine (UEMOA). Cette avancée entre dans le mécanisme de la constitution d'une monnaie unique de la Communauté Economique des Etats d'Afrique de l'Ouest (CEDEAO). Le nouvel accord de coopération monétaire entrera en vigueur après sa ratification par la France et les Etats membres de l'UEMOA. Cependant, la France avait présenté le projet de loi de ratification de cet accord au conseil des ministres du 20 Mai $2020^{27}$.

La réforme annoncée le 21 décembre 2019 s'inscrivant dans le contexte du projet de monnaie unique de la CEDEAO comporte quatre évolutions majeures à savoir :

- le changement de nom de la monnaie, les autorités de l'UMOA ont indiqué leur souhait de passer du Franc CFA à l'ECO en 2020;

- la fin de la centralisation, sur un compte d'opérations ouvert au Trésor français, de la moitié des réserves de change de la Banque centrale des États de l'Afrique de l'Ouest (BCEAO). La centralisation en France d'une partie des réserves de change sera donc supprimée dans le cadre de la réforme. A compter de la signature du nouvel accord de coopération monétaire, qui remplace l'accord qui avait été signé en 1973, la BCEAO n'aura à l'avenir plus d'obligation particulière concernant le placement de ses réserves de change. Elle sera libre de placer ses avoirs dans les actifs de son choix, en fonction des rémunérations proposées par les différents actifs.

- le retrait de la France des principales instances de décisions de la Zone. En effet, l'UEMOA exige le retrait des représentants de l'État français des instances de gouvernance dans lesquelles ils étaient présents, notamment le Conseil d'administration de la BCEAO, ainsi que la Commission bancaire. Le Comité de politique monétaire (CPM) de la BCEAO comprendra un membre indépendant nommé par le Conseil des ministres de l'UEMOA, en concertation avec le garant. Pour rappel, les accords de coopération monétaire entre l'UEMOA et la France prévoyaient jusqu'ici d'une part la centralisation d'une partie des réserves de change de la BCEAO sur un compte d'opérations, ouvert dans les écritures du

\footnotetext{
${ }^{27} \mathrm{Cf}$. le dossier législatif du projet de loi, incluant notamment le texte de l'accord et une étude d'impact.
} 
Trésor français, d'autre part la présence de la France dans un certain nombre d'instances de gouvernance. Ces deux aspects vont disparaître. La France ne nommera plus aucun représentant dans les instances de l'Union où elle était présente.

- la mise en place concomitante de mécanismes de dialogue et de surveillance des risques ad hoc (échanges d'informations, rencontres techniques en tant que de besoin, réunion des parties à la demande de l'une d'elles en cas de crise).

Cet accord du 21 décembre 2019 vient remplacer l'accord de coopération du 4 décembre 1973. Il sera complété par une convention de garantie, texte technique d'application, qui sera conclue avec la BCEAO. Avec cette réforme, le positionnement de la France évolue pour devenir celui d'un strict garant financier de la zone. Pour l'instant, le régime de change demeure inchangé, avec un maintien de la parité fixe entre l'euro et la monnaie commune de l'Union ainsi que de la garantie de convertibilité assurée par la France. Le principe de la parité fixe étant maintenu avec l'euro, 1 euro reste toujours égale à 655,957 Francs CFA et la garantie par la France de la convertibilité en euros de la monnaie émise par la BCEAO, le Franc CFA, reste illimitée. Cela sous-entend que si la BCEAO manque de disponibilités pour couvrir ses engagements en devises, elle pourra se procurer les euros nécessaires auprès de l'État français, qui demeure le garant financier de cette monnaie.

En somme la réforme des relations de coopération monétaire entre l'UEMOA et la France s'inscrit dans le contexte de la création d'une monnaie unique à l'échelle de la CEDEAO, dont certains paramètres ont été définis par les chefs d'État de la CEDEAO en juin 2019, et qui permettra d'accroître l'intégration régionale des pays d'Afrique de l'Ouest. C'est spécialement pour s'affilier au processus puis s'apprêter aux échéances futures que les autorités de l'UEMOA ont jugé nécessaire de modifier les modalités de leur coopération monétaire avec la France.

\section{Conclusion}

La Franc CFA, malgré qu'il soit passé de Franc des Colonies Françaises d'Afrique à Franc de la Communauté Financière Africaine pour la zone UEMOA et Franc de la Coopération Financière en Afrique pour la zone CEMAC, il incarne toujours l'image de la colonisation pour les pays utilisateurs. En effet, plusieurs évènements ont poussé les pays de la zone UEMOA et la France à revoir leur coopération monétaire. Ainsi, plusieurs réformes ont vu le jour et les dernières réformes s'inscrivent dans le cadre de la constitution d'une nouvelle monnaie commune de la CEDEAO, nommée Eco. Aussi, ces réformes ont transformé le rôle de la France en celui de garant financier par contre, la parité fixe avec l'euro demeure toujours pour le moment. La transformation du rôle de la France dans la gestion de la monnaie africaine a été d'une part souhaitée par les pays utilisateurs surtout par les activistes débateurs et économistes qui voyaient en cela une autre forme de colonisation et d'autre part, par les sept autres pays de la CEDEAO qui n'utilisent pas le Franc CFA, ils craignent que l'ingérence de la France porte atteinte à leur souveraineté monétaire.

Selon les conclusions de la conférence des chefs d'Etat de la Communauté Economique des Etats de l'Afrique de l'Ouest (CEDEAO) tenue le 29 juin 2019 à Abuja (Nigéria), la nouvelle monnaie 
commune Eco, devrait entrer en vigueur en 2020 dans les 15 Etats de l'espace CEDEAO mais malheureusement, nous sommes en 2021 et aucune banque centrale fédérale n'a encore vu le jour.

Nous pouvons donc dire que le Franc CFA a subi un grand progrès surtout dans ses cinq dernières années et qu'il sera bientôt abandonné au profit de la monnaie commune nommée Eco même si cela prendra un peu plus de retard que prévu. Sa mise en place prendra un retard car jusqu'à présent le choix du lieu d'implantation et la construction d'une banque centrale fédérale ne sont pas encore faits, le symbole et l'iconographie qui apparaitront sur les billets ne font pas encore consensus, d'autres questions restent encore à être élucider.

\section{BIBLIOGRAPHIES}

[1] Banque de France, "Note d'information : La zone franc", Eurosystème, 2015. PDF en ligne.

[2] Bouessel du Bourg C., "Franc CFA : l'activiste Kémi Séba arrêté à Dakar pour avoir brûlé un billet de banque", Journal jeune Afrique, 29 Août 1017. www.jeuneafrique.com.

[3] Christinaz C., "Les francs CFA brûlés par Kémi Séba enflamment les réseaux", journal le temps, 04 Septembre 2017. www.letemps.ch.

[4] Diawara M., "Cémac : pas de dévaluation du franc CFA, mais des aménagements nécessaires", Journal le Point, 24 Décembre 2016. www.lepoint.fr.

[5] Diawara M., "Dette et colonisation de l'Afrique : ces peurs que réveille la Chine", Journal le Point, 07 Mai 2019.

[6] Feindouno S., Guérineau S., Guillaumont P., Sylviane Guillaumont J. S, et Plane P., "Zone franc, croissance économique et pauvreté", Edition Ferdi, 2019.

[7] Fond Monétaire Internationale, "World Economic Outlook", 2020.

[8] Gbadamassi F., "CEDEAO : en attendant la monnaie unique...", Journal franceinfo Afrique, économie africaine, 30 juillet 2019.

[9] Gnansounou S. U. et Verdier-Chouchane A., "Mésalignement du taux de change effectif réel : Quand faudra-t-il de nouveau dévaluer le franc CFA ?", Banque Africaine de Développement, Working Paper series, $\mathrm{N}^{\circ}$ 166- Décembre 2012.

[10] Jeune Afrique, "De quoi le franc CFA est-il le nom ?", 21 Octobre 2016.

[11] Jeune Afrique et AFP, “"Eco », future monnaie unique de la CDEAO : les chefs d'État maintiennent l'objectif de 2020", Journal jeune Afrique, 2019, 02 juillet 2019.

[12] Keynes J. M., "Proposals for an International Currency Union (second draft, 18 November 1941)", in The Collected Writings of John Maynard Keynes, vol. XXV, « Activities 1940-1944 », Cambridge University Press, 1982.

[13] Le Monde et AFP, "Manifestations d'opposants au franc CFA en Afrique et en région parisienne", le Monde, 18 Septembre 2017.

[14] Licourt J., "Kémi Séba, sulfureux conseiller du gouvernement italien", le figaro, International, 04 Février 2019.

[15] -Louarn J. J., “[Entretien] Carlos Lopes: un franc CFA fluctuant «ferait sens»”, RFI, Invité Afrique, 03 Septembre 2016.

[16] Mundell R. A., "A theory of optimum currency areas", American Economic Review, Vol. 51, No. 4, 1961, pp. 657-665.

[17] Niasse Ba S., "Le Sénégal a expulsé vers la France le militant suprématiste noir Kémi Séba », Journal le Monde, Afrique, 07 Septembre 2017.

[18] Nubukpo K., "Politique monétaire et servitude volontaire. La gestion du franc CFA par la BCEAO", Politique africaine, $\mathrm{n}^{\circ} 105,2007$, p. 11.

[19] Polle B., "Franc CFA : des économistes dénoncent « la servitude monétaire »", 28 Septembre 2016.

[20] République Française, "Décret $n^{\circ} 2004-1362$ du 8 décembre 2004 portant publication de l'accord de coopération entre le Gouvernement de la République française et les gouvernements des républiques membres de l'Union monétaire ouest-africaine, signé à Dakar le 4 décembre 1973", Journal officiel de la République Française, 2004. 